\title{
Fuzzy VIKOR approach for selection of big data analyst in procurement management
}

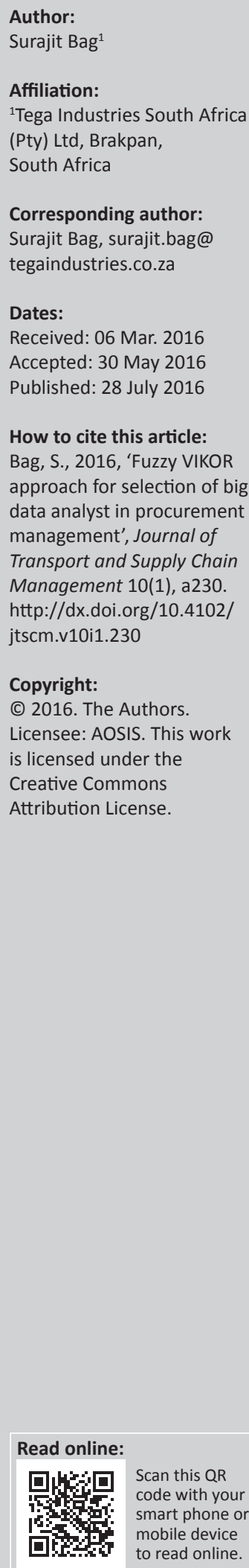

Background: Big data and predictive analysis have been hailed as the fourth paradigm of science. Big data and analytics are critical to the future of business sustainability. The demand for data scientists is increasing with the dynamic nature of businesses, thus making it indispensable to manage big data, derive meaningful results and interpret management decisions.

Objectives: The purpose of this study was to provide a brief conceptual review of big data and analytics and further illustrate the use of a multicriteria decision-making technique in selecting the right skilled candidate for big data and analytics in procurement management.

Method: It is important for firms to select and recruit the right data analyst, both in terms of skills sets and scope of analysis. The nature of such a problem is complex and multicriteria decision-making, which deals with both qualitative and quantitative factors. In the current study, an application of the Fuzzy VIsekriterijumska optimizacija i KOmpromisno Resenje (VIKOR) method was used to solve the big data analyst selection problem.

Results: From this study, it was identified that Technical knowledge (C1), Intellectual curiosity (C4) and Business acumen (C5) are the strongest influential criteria and must be present in the candidate for the big data and analytics job.

Conclusion: Fuzzy VIKOR is the perfect technique in this kind of multiple criteria decisionmaking problematic scenario. This study will assist human resource managers and procurement managers in selecting the right workforce for big data analytics.

\section{Introduction}

Big data and business analytics has been the pressing call of the day. Earlier big data research was limited to the information science researchers, but recently management scientists have started to study the connections of big data with business performance. There are several studies on big data where researchers have explored its connection with Finance and auditing (Brown-Liburd, Issa \& Lombardi 2015; Cao, Chychyla \& Stewart 2015; Griffin \& Wright 2015; Warren, Moffitt \& Byrnes 2015; Yoon, Hoogduin \& Zhang 2015), Communications (Davis 2014), Healthcare (Ebenezer \& Durga 2015), Marketing (Fulgoni 2013; Nunan \& Di Domenico 2013) and Supply chain management (Rozados \& Tjahjono 2014; Waller \& Fawcett 2013a, 2013b). The purpose of this study is to provide a brief conceptual review of big data and analytics. This study largely illustrates the use of an innovative multicriteria decision-making Fuzzy VIsekriterijumska optimizacija i KOmpromisno Resenje (VIKOR) technique in selecting the right skilled candidate for big data and analytics in procurement management function. The next section presents the literature review followed by research methods, data analysis and results, discussion, conclusion, limitations and future research directions.

\section{Literature review}

The literature review search strategies involved conducting a search within the time frame ranging from 2011 to 2016, which was considered to be the representative period covering the emergence of 'big data', with a comprehensive search using the word 'big data' within the databases: EBSCO and Business Source Complete. First, the author discusses the advance computing system on which rest the concept of big data and predictive analysis.

An advance computing system embodies the hardware, software and algorithms. Advance computing is defined by multiple petaflops supercomputing systems and cloud data centres with many petabytes of secondary storage. Tools such as Pig provide a high-level programming model. Other tools such as Mahout enable classification, recommendation and prediction via supervised and unsupervised learning (Reed \& Dongara 2015). 
For the last 2 or 3 years, the field of big data has emerged as the new frontier in the wide spectrum of IT-enabled innovations and opportunities. Big data is about massive amounts of observational data, of different types, supporting different types of decisions and decision time frames. Big data has been defined by the 4 V's - volume, velocity, variety and veracity. Volume: terabytes or even petabytes of data are generated. Velocity: speed at which data are generated, delivered and processed. Variety: big data comes in all forms - structured, semi-structured and unstructured. Veracity: uncertainty of data. Analytics refers to the upper stages of the hierarchy: the generation of knowledge and intelligence to support decision-making and strategic objectives (Goes 2014).

Big data and analytics are critical to the future of advanced computing and scientific discovery. Data generation capabilities in most scientific domains are growing more rapidly than computing abilities, causing these domains to become data intensive (Reed \& Dongara 2015). Data analytic clusters are typically based on commodity Ethernet networks and local storage, with cost and capacity being the primary optimisation criteria. Large-scale data preservation and sustainability within and across disciplines, metadata creation and multidisciplinary fusion, and digital privacy and security define the frontiers of big data (Reed \& Dongara 2015). 'As scientific research increasingly depends on both high-speed computing and data analytics, the potential interoperability and scaling convergence of these two ecosystems is crucial to the future' (Reed \& Dongara 2015:56-68).

The areas that are identified as big impact areas of big data research are e-commerce and market intelligence, e-government and politics, science and technology, smart health and well-being, and security and public safety (Goes 2014).

Companies that use data-directed decision-making enjoy a $5 \%-6 \%$ boost in productivity. It requires understanding how and when to use the data in making crucial decisions. Competitive advantage can be greatly improved by leveraging the right data. Data-driven decisions tend to be better decisions (Alles 2015).

Creating value for big data is a multistep process: acquisition, information extraction and cleaning, data integration, modelling and analysis, and interpretation and deployment. Many discussions on big data focus on only one or two steps, ignoring the rest. Huge rewards wait for those who use big data correctly (Jagadish et al. 2014).

A study by Huang and Huang (2015) revealed that big data focuses on aggregating multiple data sets, analyses them, and looks for meaningful patterns. The last mile of big data is where the value is created, opinions are formed and insights are shared, and actions are made. Big data is composed of small data pieces joined together. Analysts generate value from the data, but data do not generate the value itself.
Which data should be picked, what data should be integrated or what data should be collected are all managed by the analyst. The analyst is the key position of the whole process (Huang \& Huang 2015). Techniques such as statistics, econometrics, machine learning, computational, linguistics, optimisation and simulation are essential for becoming a big data and analytics expert (Goes 2014; Jeong \& Imran 2014).

However, major limitations potentially related to information processing information in a big data environment include: information overload, information relevance, pattern recognition and ambiguity. The nature of big data is unstructured (emails, company blogs), which makes it difficult to choose relevant data. Predictive analytics identifies meaningful patterns of data to foresee unknown future events using the insights of big data. Predictive analysis can be applied to ambiguous and highly subjective judgements, to model relationships amongst various relevant factors (Brown-Liburd et al. 2015). Also, the nature of big data qualities of volume, velocity, variety and veracity contributes to the creation of the big data gaps such as data consistency, data integrity, data identification, data aggregation and data confidentiality (Zhang, Yang \& Appelbaum 2015).

The associated challenges are that today's advanced computing and data analysis systems consume megawatts of power, necessitating new infrastructure approaches and operating models, low-power designs, cooling approaches, energy accountability and operational efficiencies (Reed \& Dongara 2015).

Now coming to the technology side of big data where Hadoop, an open source platform, is the most widely applied technology for managing storage and access. Hadoop is a challenge for medium- and small-sized businesses, as the application requires expertise and experience not widely available. Moreover, finding the right talent to analyse big data is perhaps the greatest challenge, as required skills are neither simple nor solely technology oriented. In business, challenges are availability of data scientists (analysts, statisticians) and data mining (storing, interlinking, processing) (Kim, Trimi \& Chung 2014). Now, let us understand the link between big data and supply management. There are several earlier published articles where this connection has been explained. Avery (2016) echoed that big data and extreme negotiations were the topic of presentation recently at the Institute for Supply Management, Greater Boston. McGovern (2014) found that most of the procurement organisations excel at leveraging analytics. Suppliers are critical to any business. Technology solutions that can effectively and comprehensively consolidate and connect all forms of supplier data across a global organisation are gaining popularity. Another article by Mistry (2016) discussed the challenges and opportunities related to procurement in the age of big data. It is said that top procurement managers focused more on big data and analytics. They intend to make spend analysis, contract management, supplier management and performance management analytics a determining factor of their future performance. But, there is a need to impart training and educate procurement teams in order to develop the backbone of companies' integrated procurement analytics approaches. 
The current study is motivated by the past studies conducted by Avery (2016), Mistry (2016), Kim et al. 2014 and McGovern (2014), where indications of skill gaps, training, education and development related to big data and data analysis in supply management have been discussed. The objective of the present study is to provide a brief conceptual review of big data and analytics; this study largely illustrates the use of an innovative multicriteria decision-making approach called Fuzzy VIKOR in selecting the right skilled candidate for big data and analytics in procurement management. The next section presents the research method used in the current study.

\section{Research methods}

The human language is filled with imprecision, subjectivities and vagueness when used tojudge, describe and communicate information. In view of this, Zadeh (1978) introduced the fuzzy set theory to model human judgements (Afful-Dadzie, Nabareseh \& Oplatková 2014). Fuzzy logic has been extended to almost all other Multi criteria Decision Making (MCDM) techniques such as Analytic Hierarchy Process (AHP), Analytic Network Process (ANP), Elimination and Choice Expressing Reality (ELECTRE), Grey Relational Analysis (GRA), Preference Ranking Organization Method for Enrichment Evaluation (PROMETHEE), Technique for Order Preference by Similarity to Ideal Solution (TOPSIS), Weighted Product Model and VIKOR (Afful-Dadzie et al. 2014). Based on the research objectives, the author found Fuzzy VIKOR to be suitable for the current study. The next section presents the overview of Fuzzy VIKOR method.

\section{Fuzzy VIKOR method}

Professor Serafim Opricovic had developed the concept of VIKOR in his PhD dissertation in 1979, and an application was published in the water resources research journal (Duckstein \& Opricovic 1980). The name VIKOR appeared in 1990 from Serbian: VIseKriterijumska Optimizacija I Kompromisno Resenje, which means: multicriteria optimisation and compromise solution, with pronunciation: VIKOR (Opricovic 1990). The real applications were presented in the study, which appeared in 1998 (Opricovic 1998). The concept of fuzzy set theory and VIKOR method has been combined to develop the Fuzzy VIKOR technique. This technique can be applied to find the best compromise solution under the situation of multiperson multicriteria decision-making problem. Generally, decision-making problems deal with certain alternatives which can be ranked with respect to different criteria. Ratings of the alternatives and the weights of each criterion are the two most significant data, which can affect the results of decision-making problems (Samantra 2012). Therefore, the proposed methodology has been used here to calculate the definite weight of criteria and ranking of the alternatives. In this article, the importance of weights of various criteria and ratings of qualitative criteria are measured as linguistic variables because linguistic assessment can only have the capability to approximate the subjective judgement through a decision-maker's opinion. Moreover, linear triangular membership functions are considered for capturing the vagueness of these linguistic assessments (Samantra 2012).

\section{Data analysis and results}

The steps of Fuzzy VIKOR have been followed as suggested by Opricovic and Tzeng $(2004,2007)$ and Afful-Dadzie et al. (2014). In the current study, the firm intends to select the analyst having expertise in big data and analytics and specifically knows the application in purchasing and supply chain management. Here, three candidates (X1, X2 and X3) have been evaluated by three decision makers (Y1, Y2 and Y3) based on eight skill sets such as Technical knowledge (C1), Time management (C2), Flexibility (C3), Intellectual curiosity (C4), Business acumen (C5), Strong interpersonal skills (C6), Demand planning (C7) and Supplier relationship management (C8).

\section{Step 1: Determining linguistic variables}

The first step in the Fuzzy VIKOR method is to determine the linguistic variables, the criteria for selecting the big data and analytics expert for purchasing and supply management function. Linguistic terms transformed into fuzzy numbers are used by the experts to rate each linguistic variable. Linguistic terms are qualitative words or phrases of a natural language that reflect the subjective view of an expert about the criteria per each alternative under consideration. In this study, triangular fuzzy numbers are used as shown in Tables 1 and 3, respectively, to capture the ratings of the criteria and alternatives on a scale of $0-1$ (Afful-Dadzie et al. 2014).

\section{Step 2: Determining the importance of weight of criteria}

The second step in the Fuzzy VIKOR process offers evaluators the chance to choose by rating the most important criteria for the evaluation guided by the linguistic terms in Table 1. The linguistic preferences for the three decision makers concerning the importance attached to each criterion are shown in Table 2. Also, the linguistic variables for the rating of alternatives are presented in Table 3.

\section{Step 3: Constructing the Best Non Fuzzy Performance value}

The graded mean integration method is used to aggregate the three decision makers' opinions regarding the importance of weightings of each criterion. The result of such aggregation is shown in Table 4. To determine the importance of each criterion by ranking, the fuzzy numbers are defuzzified (Afful-Dadzie et al. 2014). The article uses the centre of area method in computing the Best Non-Fuzzy Performance value (BNP) to rank the order of importance of each criterion (Afful-Dadzie et al. 2014). The BNP value of the fuzzy number $\mathrm{W}_{\mathrm{k}}=\left(\mathrm{L}_{\mathrm{wk}}, \mathrm{M}_{\mathrm{wk}}, \mathrm{U}_{\mathrm{wk}}\right)$ is calculated using Equation 1 as follows:

$\mathrm{BNP}_{\mathrm{wk}}=\mathrm{L}_{\mathrm{wk}}+\left[\left(\mathrm{U}_{\mathrm{wk}}-\mathrm{L}_{\mathrm{wk}}\right)+\left(\mathrm{M}_{\mathrm{wk}}-\mathrm{L}_{\mathrm{wk}}\right)\right] / 3$

[Eqn 1]

By the BNP value computation, the major influential criteria out of the eight are $\mathrm{C} 1, \mathrm{C} 4$ and $\mathrm{C} 5$ with a rank of 1 , 
TABLE 1: Linguistic variables for the importance of weight of criteria.

\begin{tabular}{lc}
\hline Linguistic terms & Corresponding fuzzy numbers \\
\hline Very low (VL) & $(0.0,0.0,0.1)$ \\
Low (L) & $(0.0,0.1,0.3)$ \\
Medium low (ML) & $(0.1,0.3,0.5)$ \\
Medium (M) & $(0.3,0.5,0.7)$ \\
Medium high (MH) & $(0.5,0.7,0.9)$ \\
High (H) & $(0.7,0.9,1.0)$ \\
Very high (VH) & $(0.9,1.0,1.0)$ \\
\hline
\end{tabular}

TABLE 2: The importance weight of the criteria.

\begin{tabular}{llll}
\hline Criteria and alternatives & Y1 & Y2 & Y3 \\
\hline C1 & VH & VH & VH \\
C2 & VH & H & MH \\
C3 & H & H & MH \\
C4 & VH & VH & VH \\
C5 & VH & VH & VH \\
C6 & $H$ & $V H$ & VH \\
C7 & $H$ & $H$ & VH \\
C8 & $H$ & MH & H \\
\hline
\end{tabular}

$\mathrm{H}$, high; $\mathrm{MH}$, medium high; $\mathrm{VH}$, very high.

TABLE 3: Linguistic variables for the rating of alternatives.

\begin{tabular}{ll}
\hline Linguistic terms & Corresponding fuzzy numbers \\
\hline Very poor (VP) & $(0.0,0.0,1.0)$ \\
Poor (P) & $(0.0,1.0,3.0)$ \\
Medium poor (MP) & $(1.0,3.0,5.0)$ \\
Fair (F) & $(3.0,5.0,7.0)$ \\
Medium good (MG) & $(5.0,7.0,9.0)$ \\
Good (G) & $(7.0,9.0,10)$ \\
Very good (VG) & $(9.0,10,10)$ \\
\hline
\end{tabular}

TABLE 4: Aggregated importance of weight of the criteria.

\begin{tabular}{llll}
\hline Criteria & TFN & BNP & Rank \\
\hline C1 & $(0.9,1,1)$ & 0.96 & 1 \\
C2 & $(0.7,0.86,0.96)$ & 0.84 & 4 \\
C3 & $(0.63,0.83,0.96)$ & 0.80 & 5 \\
C4 & $(0.9,1,1)$ & 0.96 & 1 \\
C5 & $(0.9,1,1)$ & 0.96 & 1 \\
C6 & $(0.83,0.96,1)$ & 0.93 & 2 \\
C7 & $(0.76,0.93,1)$ & 0.89 & 3 \\
C8 & $(0.63,0.83,0.96)$ & 0.80 & 5 \\
\hline
\end{tabular}

BNP, Best Non-Fuzzy Performance value.

and C6, C7 and C2 with a rank of 2, 3 and 4, respectively. The least important criterion would be $\mathrm{C} 8$ with a rank of 5 .

\section{Step 4: Constructing the fuzzy rating matrix}

Here, the decision makers rate the various candidates using linguistic terms in Table 3. The fuzzy rating of three decision makers on eight skills is presented in Table 5 .

\section{Step 5: Constructing aggregated triangular fuzzy number decision matrix}

Table 6 demonstrates the ratings of evaluators which have been aggregated using the following equation:

$\mathrm{a}_{\mathrm{ij}}=\min _{\mathrm{k}}\left\{\mathrm{a}_{\mathrm{ij}}^{\mathrm{k}}\right\}$,

$\mathrm{b}_{\mathrm{ij}}=1 / \mathrm{k} \sum_{\mathrm{k}=1}^{\mathrm{k}} \mathrm{b}_{\mathrm{ij}}$
TABLE 5: The fuzzy rating of three decision makers on eight skills.

\begin{tabular}{|c|c|c|c|c|}
\hline \multirow[b]{2}{*}{ Criteria } & \multirow[b]{2}{*}{ Candidates } & \multicolumn{3}{|c|}{ Decision makers } \\
\hline & & $\mathrm{Y1}$ & Y2 & Y3 \\
\hline \multirow[t]{3}{*}{ C1 } & $\mathrm{X} 1$ & G & MG & MG \\
\hline & $\mathrm{X} 2$ & G & VG & VG \\
\hline & X3 & MG & G & MG \\
\hline \multirow[t]{3}{*}{$\mathrm{C} 2$} & $\mathrm{X} 1$ & G & G & G \\
\hline & $\mathrm{X} 2$ & G & VG & G \\
\hline & X3 & $\mathrm{F}$ & MG & $\mathrm{F}$ \\
\hline \multirow[t]{3}{*}{ C3 } & $\mathrm{X} 1$ & G & G & $\mathrm{F}$ \\
\hline & $\mathrm{X} 2$ & VG & VG & G \\
\hline & X3 & MG & MG & G \\
\hline \multirow[t]{3}{*}{ C4 } & $\mathrm{X} 1$ & VG & VG & VG \\
\hline & $\mathrm{X} 2$ & VG & G & VG \\
\hline & X3 & MG & $F$ & MG \\
\hline \multirow[t]{3}{*}{ C5 } & $\mathrm{X} 1$ & VG & G & G \\
\hline & $\mathrm{X} 2$ & G & VG & G \\
\hline & X3 & G & G & G \\
\hline \multirow[t]{3}{*}{ C6 } & $\mathrm{X} 1$ & G & G & G \\
\hline & $\mathrm{X} 2$ & VG & VG & G \\
\hline & X3 & G & G & VG \\
\hline \multirow[t]{3}{*}{$\mathrm{C7}$} & $\mathrm{X} 1$ & MG & G & G \\
\hline & $\mathrm{X} 2$ & G & $\mathrm{F}$ & G \\
\hline & $\mathrm{X} 3$ & G & $\mathrm{F}$ & $\mathrm{F}$ \\
\hline \multirow[t]{3}{*}{$\mathrm{C} 8$} & $\mathrm{X} 1$ & G & G & VG \\
\hline & $\mathrm{X} 2$ & MG & $\mathrm{F}$ & MG \\
\hline & X3 & $\mathrm{F}$ & $\mathrm{MP}$ & $\mathrm{F}$ \\
\hline
\end{tabular}

F, fair; G, good; $M G$, medium good; $M P$, medium poor; $V G$, very good.

$c_{i j}=\max _{k}\left\{c_{i j}^{k}\right\}, i=1,2, \ldots m ; j=1,2, \ldots n$

[Eqn 4]

\section{Step 6: Fuzzy best value ( $\left.\mathrm{ff}^{*}\right)$ and fuzzy worst value ( $\sim$ fj-)}

Here, the fuzzy best and fuzzy worst values for the evaluation criteria were determined. The result of this process is shown in Table 7.

\section{Step 7: Computing separation measures $\sim$ Si and $\sim$ Ri}

The separation measures of $\sim S_{i}$ and $\sim R_{i}$ of alternative $A_{i}$ from the fuzzy best and worst values, respectively, are computed and presented in Table 8.

\section{Step 8: Computing the value of Qi}

$$
\begin{aligned}
& \mathrm{Q} 1=(-0.65,0.05,0.72) \\
& \mathrm{Q} 2=(-0.63,0.00,0.63) \\
& \mathrm{Q} 3=(-0.47,0.29,1.00)
\end{aligned}
$$

\section{Step 9: Defuzifying values of $\sim \mathrm{Si}, \sim \mathrm{Ri}$ and $\sim \mathrm{Qi}$}

The defuzzification process converts $\sim S_{i^{\prime}} \sim R_{i}$ and $\sim Q_{i}$ into crisp numbers $S, R$ and $Q$, respectively. The results are shown in Table 9.

\section{Step 10: Ranking the alternatives}

The crisp value of the alternatives for $Q$ is ranked from the smallest value to the highest value. The alternatives are ranked as shown in Table 9 (Afful-Dadzie et al. 2014). 
TABLE 6: Aggregated triangular fuzzy number decision matrix.

\begin{tabular}{|c|c|c|c|c|c|c|c|c|}
\hline$x$ & C1 & $\mathrm{C2}$ & C3 & $\mathrm{C} 4$ & C5 & $\mathrm{C6}$ & C7 & $\mathrm{C} 8$ \\
\hline$\overline{x 1}$ & $(5.66,7.66,9.33)$ & $(7,9,10)$ & $(5.66,7.66,9)$ & $(9.0,10,10)$ & $(7.66,9.33,10)$ & $(7.0,9.0,10)$ & $(6.33,8.33,9.66)$ & $(7.66,9.33,10)$ \\
\hline $\mathrm{x} 2$ & $(8.33,9.66,10)$ & $(7.66,9.33,10)$ & $(8.33,9.66,10)$ & $(8.33,9.66,10)$ & $(7.66,9.33,10)$ & $(8.33,9.66,10)$ & $(5.66,7.66,9)$ & $(4.33,6.33,8.33)$ \\
\hline $\mathrm{x} 3$ & $(5.66,7.66,9.33)$ & $(3.66,5.66,7.66)$ & $(5.66,7.66,9.33)$ & $(4.33,6.33,8.33)$ & $(7.0,9.0,10)$ & $(7.66,9.33,10)$ & $(4.33,6.33,8)$ & $(2.33,4.33,6.33)$ \\
\hline
\end{tabular}

TABLE 7: Fuzzy best value $(\sim f j *)$ and fuzzy worst value ( $\sim \mathrm{fj}-)$.

\begin{tabular}{|c|c|c|c|c|c|c|c|c|}
\hline Best and worst value & C1 & $\mathrm{C2}$ & C3 & $\mathrm{C4}$ & C5 & C6 & C7 & $\mathrm{C} 8$ \\
\hline$f_{j}^{*}$ & $(8.33,9.66,10)$ & $(7,9,10)$ & $(8.33,9.66,10)$ & $(9.0,10,10)$ & $(7.66,9.33,10)$ & $(8.33,9.66,10)$ & $(6.33,8.33,9.66)$ & $(7.66,9.33,10)$ \\
\hline$f_{-}-$ & $(5.66,7.66,9.33)$ & $(3.66,5.66,7.66)$ & $(5.66,7.66,9)$ & $(4.33,6.33,8.33)$ & $(7.0,9.0,10)$ & $(7.0,9.0,10)$ & $(4.33,6.33,8)$ & $(2.33,4.33,6.33)$ \\
\hline
\end{tabular}

TABLE 8: Index $\sim$ Si and $\sim \mathrm{Ri}$.

\begin{tabular}{llll}
\hline Separation measures & X1 & X2 & X3 \\
\hline$\sim S_{i}$ & $(-2.6,1.09,5.22)$ & $(-2.6,0.44,4.25)$ & $(-0.89,3.60,8.18)$ \\
$\sim R_{i}$ & $(-0.70,0,0.78)$ & $(-0.70,0,0.78)$ & $(-0.70,0.11,1)$ \\
\hline
\end{tabular}

TABLE 9: Defuzzified values of $S, R$ and $Q$.

\begin{tabular}{lccc}
\hline Defuzzified values & X1 & X2 & X3 \\
\hline $\mathrm{Q}$ & 0.04 & 0.00 & 0.27 \\
$\mathrm{~S}$ & 1.23 & 0.69 & 3.63 \\
$\mathrm{R}$ & 0.02 & 0.02 & 0.13 \\
\hline
\end{tabular}

TABLE 10: Rank for alternatives

\begin{tabular}{lccc}
\hline Alternatives & X1 & X2 & X3 \\
\hline Results (Q) & 0.04 & 0.01 & 0.27 \\
Rank & 2 & 1 & 3 \\
\hline
\end{tabular}

As stated above, the smaller $\mathrm{Q}_{\mathrm{i}}$ implies the better performance of a candidate. Hence, $\mathrm{X} 2$ is given the precedence over $\mathrm{X} 1$ and $\mathrm{X} 3$ in that order.

\section{Step 11: Proposing a compromise solution}

In Table 10, the best ranked candidate is X2 which happens to be the best compromise solution (Afful-Dadzie et al. 2014).

\section{Discussion}

It is found that Technical knowledge (C1), Intellectual curiosity (C4) and Business acumen (C5) are the strongest influential criteria and must be present in the candidate for the big data and analytics job. Technical knowledge such as basic statistics, understanding of machine learning, querying language (SQL, Hive, Pig), scripting language (Python, Matlab), statistical language (R, SAS, SPSS) and spreadsheet (Excel) are essential. Intellectual curiosity involves rationale, logical approach to problems, methodological and problem solving. Business acumen is also essential for understanding the market supply and demand patterns and improves the firm's profitability. The problem solution shows that out of three candidates, the second candidate possesses the right skill sets and is the best candidate for the job. Fuzzy VIKOR technique eliminates the limitations of simple VIKOR technique and provides a better quality of output.

\section{Conclusion}

The current study provides a brief conceptual background of big data and business analytics and further identified the research gaps. It was found that big data and analytics play an important role in procurement management.
Procurement involves contract management, budget, request for quote, supplier relationship management, spend analysis, inventory management and cost savings. If the big data related to these activities can be managed properly, then the annual savings leakage can be stopped and better business decisions can be made. However, most of the organisations lack the right workforce and therefore arises the need to either educate and train existing workforce or select and recruit the big data and analytics expert. Keeping the research objectives and research questions in mind, here Fuzzy VIKOR has been applied to select the right candidate for big data and analytics job in purchasing function. From the study, it has been identified that Technical knowledge (C1), Intellectual curiosity (C4) and Business acumen (C5) are the strongest influential criteria and must be present in the candidate for the big data and analytics job. Lastly, the problem solution shows that out of three candidates, the second candidate possesses the right skill sets and is the best candidate for the job.

\section{Managerial implications}

The study provides rich insights for chief procurement officers who intend to hire the right candidate for big data and analytics job in procurement management function. Firstly, the study brings forward the essential skill sets which must be evaluated during interview. Secondly, organisations can also invest in training programs to build these skill sets within the existing workforce. Ultimately, it is clear that big data and analytics are the pressing call of the day for business sustainability, and organisations must look for technical solutions as well as right workforce for managing big data.

\section{Limitations and future research directions}

The limitations of the current study involve the human intervention in the process, which is basically the subjective judgement conducted through a decision-maker's opinion. This model can be compared using alternate MCDM techniques such as TOPSIS method to compare the results obtained in the current study.

\section{Acknowledgements}

The author wishes to thank all the reviewers for their helpful and valuable comments on this article. The author is also thankful to his wife for her encouragement in developing this article. 


\section{Competing interests}

The author declares that there are no financial or personal relationships which may have inappropriately influenced him in writing this article.

\section{References}

Afful-Dadzie, E., Nabareseh, S. \& Oplatková, Z.K., 2014, 'Fuzzy VIKOR approach: Evaluating quality of internet health information', Annals of Computer Science and Information Systems 2, 183-190. http://dx.doi.org/10.15439/2014F203

Alles, M.G., 2015, 'Drivers of the use and facilitators and obstacles of the evolution of big data by the audit profession', Accounting Horizons 29(2), 439-449. http://dx. doi.org/10.2308/acch-51067

Avery, S., 2016, 'Big data, extreme negotiations and supply management', viewed 01 December 2015, from http://www.mypurchasingcenter.com/purchasing/ industry-articles/big-data-extreme-negotiations-and-supply-management/

Brown-Liburd, H., Issa, H. \& Lombardi, D.R., 2015, 'Behavioral implications of big data's impact on audit judgment and decision making and future research directions', Accounting Horizons 29(2), 451-468. http://dx.doi.org/10.2308/acch51023

Cao, M., Chychyla, R. \& Stewart, T., 2015, 'Big data analytics in financial statement audits', Accounting Horizons 29(2), 423-429. http://dx.doi.org/10.2308/acch51068

Davis, C.K., 2014, 'Beyond data and analysis', Communications of the ACM 57(6), 39-41. http://dx.doi.org/10.1145/2602326

Duckstein, L. \& Opricovic, S., 1980, 'Multiobjective optimization in river basin development', Water Resources Research 16(1), 14-20. http://dx.doi. org/10.1029/WR016i001p00014

Ebenezer, J.G.A. \& Durga, S., 2015, 'Big data analytics in healthcare: A survey', Journal of Engineering \& Applied Sciences 10(8), 3645-3650.

Fulgoni, G., 2013, 'Big data: Friend or foe of digital advertising? Five ways marketers should use digital big data to their advantage', Journal of Advertising Research 53(4), 372-376. http://dx.doi.org/10.2501/JAR-53-4-372-376

Goes, P.B., 2014, 'Big data and IS research', MIS Quarterly 38(3), 3-8.

Griffin, P.A. \& Wright, A.M., 2015, 'Commentaries on big data's importance for accounting and auditing', Accounting Horizons 29(2), 377-379. http://dx.doi. org/10.2308/acch-51066

Huang, P.C. \& Huang, P.S., 2015, 'When big data gets small', International Journal of Organizational Innovation 8(2), 100-117.

Jagadish, H.V., Gehrke, J., Labrinidis, A., Papakonstantinou, Y., Patel, J.M. Ramakrishnan, R. et al., 2014, 'Big data and its technical challenges', Communications of the ACM 57(7), 86-94. http://dx.doi.org/10.1145/2611567

Jeong, S.R. \& Imran, G., 2014, 'Semantic computing for big data: Approaches, tools, and emerging directions', KSII Transactions on Internet \& Information Systems 8(6), 2022-2042. http://dx.doi.org/10.3837/tiis.2014.06.012
Kim, G.H., Trimi, S. \& Chung, J.H., 2014, 'Big-data applications in the government sector', Communications of the ACM 57(3), 78-85. http://dx.doi.org/10.1145/ 2500873

McGovern, M., 2014, 'Big value for procurement in big data', viewed 03 December 2015, from http://www.mypurchasingcenter.com/technology/technology2015, from http://www.mypurchasin

Mistry, R., 2016, 'Procurement in the age of big data challenges and opportunities', viewed 01 December 2015, from http://www.mypurchasingcenter.com/ technology/technology-articles/procurement-age-big-data-challenges-andopportunities

Nunan, D. \& Di Domenico, M., 2013, 'Market research and the ethics of big data', International Journal of Market Research 55(4), 505-520. http://dx.doi. org/10.2501/IJMR-2013-015

Opricovic, S., 1990, 'Programski paket VIKOR za visekriterijumsko kompromisno rangiranje', SYMOPIS.

Opricovic, S., 1998, Multicriteria optimization in civil engineering (in Serbian), Faculty of Civil Engineering, Belgrade, 302 p. ISBN 86-80049-82-4

Opricovic, S. \& Tzeng, G.H., 2004, 'The Compromise solution by MCDM methods: A comparative analysis of VIKOR and TOPSIS', European Journal of Operationa Research 156(2), 445-455. http://dx.doi.org/10.1016/S0377-2217(03)00020-1

Opricovic, S. \& Tzeng, G.H., 2007, 'Extended VIKOR method in comparison with outranking methods', European Journal of Operational Research 178(2), 514-529. http://dx.doi.org/10.1016/j.ejor.2006.01.020

Reed, D.A. \& Dongarra, J., 2015, 'Exascale computing and big data', Communications of the ACM 58(7), 56-68. http://dx.doi.org/10.1145/2699414

Rozados, I.V. \& Tjahjono, B., 2014, 'Big data analytics in supply chain management: Trends and related research', 6th International Conference on Operations and Supply Chain Management, Bali, 2014.

Samantra, C., 2012, 'Decision-making in fuzzy environment', Doctoral dissertation, National Institute of Technology Rourkela 769008, India.

Waller, M.A. \& Fawcett, S.E., 2013a, 'Click here for a data scientist: Big data, predictive analytics, and theory development in the era of a maker movement supply chain', Journal of Business Logistics 34(4), 249-252. http://dx.doi.org/10.1111/jbl.12024

Waller, M.A. \& Fawcett, S.E., 2013b, 'Data science, predictive analytics, and big data: A revolution that will transform supply chain design and management', Journal of Business Logistics 34(2), 77-84. http://dx.doi.org/10.1111/jbl.12010

Warren, Jr, J.D., Moffitt, K.C. \& Byrnes, P., 2015, 'How big data will change accounting', Accounting Horizons 29(2), 381-396. http://dx.doi.org/10.2308/acch-51069

Yoon, K., Hoogduin, L.A. \& Zhang, L., 2015, 'Big data as complementary audit evidence', Accounting Horizons 29(2), 431-438. http://dx.doi.org/10.2308/acch51076

Zadeh, L.A., 1978, 'Fuzzy sets as a basis for a theory of possibility', Fuzzy Sets and Systems 1(1), 3-28. http://dx.doi.org/10.1016/0165-0114(78)90029-5

Zhang, J., Yang, X. \& Appelbaum, D., 2015, 'Toward effective big data analysis in continuous auditing', Accounting Horizons 29(2), 469-476. http://dx.doi. org/10.2308/acch-51070 